\title{
Confined harmonically interacting spin-polarized fermions
}

\author{
F. Brosens and J. T. Devreese* \\ Departement Natuurkunde, Universitaire Instelling Antwerpen, Universiteitsplein 1, B-2610 Antwerpen, Belgium \\ L. F. Lemmens \\ Departement Natuurkunde, Rijksuniversitair Centrum te Antwerpen, Universiteit te Antwerpen, Groenenborgerlaan 171, \\ B-2020 Antwerpen, Belgium \\ (Received 14 October 1997)
}

\begin{abstract}
The thermodynamical properties are calculated for a three-dimensional model of $N$ harmonically interacting spin-polarized fermions in a parabolic potential well. The obtained dependences of the chemical potential and of the internal energy on the complete range of the temperature and of the number of particles turn out to obey a scaling law, similar to the scaling from the continuum approximation for the density of states. The calculational technique is based on our path-integral approach of symmetrized density matrices for identical particles in a parabolic confining well. [S1063-651X(98)03504-1]
\end{abstract}

PACS number(s): 05.30.-d, 03.75.Fi, 32.80.Pj

\section{INTRODUCTION}

In two previous papers the present authors extended the method of symmetrized density matrices to systems confined in a parabolic well [1] and used this method to obtain expressions for the density and the pair-correlation function [2]. The evaluation of the internal energy, the specific heat, the moment of inertia $[3,4]$, the density, and the static response functions was performed for bosons, inspired by the recently observed Bose-Einstein condensation [5-7] and the theoretical work around this phenomenon using other methods [8-14]. In both Refs. [1] and [2] the general expressions for most of the quantities mentioned above are also given for Fermi-Dirac statistics. In the present paper a method is presented to explicitly evaluate the thermodynamic quantities for spin-polarized fermions. The model is a parabolic well containing $N$ fermions, all in the same spin state and interacting through a harmonic two-body potential that may be either attractive or repulsive.

A quantum dot would be a physical system that could be described by such a model if also a magnetic field were taken into account to freeze away the opposite spin states. When no magnetic field is taken into account two spin states (spin up and spin down) should be present in the model. Recently [15], the investigation of confined fermions in the same experimental configuration used for the Bose alkali metals was proposed. The Thomas-Fermi approximation [16] was used to study the spatial distribution of these trapped fermion systems. In order to analyze these more physically relevant models in the future, we first develop in the present paper the basic techniques required for spin-polarized fermions. The model also has some importance in itself because it can be used to test new approaches to Monte Carlo simulations of interacting fermions such as many-body diffusion [17-19].

\footnotetext{
*Also at Rijksuniversitair Centrum te Antwerpen, Universiteit Antwerpen, Groenenborgerlaan 171, B-2020 Antwerpen, Belgium and Technische Universiteit Eindhoven, NL 5600MB Eindhoven, The Netherlands.
}

The paper is organized as follows. In Sec. II we collect the expressions from [1] and [2] for the fermion case. In Sec. III we show how the chemical potential, the free energy, and the internal energy can be obtained for a given number of fermions as a function of the temperature. Subsequently the low-temperature limit is considered and the ground-state energy is evaluated in Sec. IV. In Sec. V a discussion and the conclusions are given.

\section{FERMION OSCILLATORS}

In this section the basic formulas that have been derived in the path-integral treatment of [1] and [2] are summarized and rewritten in such a way that they are more appropriate for dealing with fermions, in particular for the numerical treatment. Before doing so, it is instructive to point out where the numerical accuracy problems are coming from. Having pinpointed their origin, a method is proposed to accurately evaluate the relevant thermodynamic quantities.

\section{A. Summary of previous results}

We consider a model of $N$ fermions with parallel spin in a harmonic confinement potential and with a quadratic interparticle interaction. The one-body potential energy $V_{1}$ and the two-body potential energy $V_{2}$ of the model system are given by

$$
\begin{gathered}
V=V_{1}+V_{2}, \quad V_{1}=\frac{m \Omega^{2}}{2} \sum_{j=1}^{N} \mathbf{r}_{j}^{2}, \\
V_{2}=-\frac{m \omega^{2}}{4} \sum_{j, l=1}^{N}\left(\mathbf{r}_{j}-\mathbf{r}_{l}\right)^{2} .
\end{gathered}
$$

The two-body interaction is assumed to be repulsive; replacing $-\omega^{2}$ by $\omega^{2}$ in $V_{2}$ gives the case of attraction. In each dimension we found one degree of freedom (the center of mass) with frequency $\Omega$ and $N-1$ degrees of freedom with frequency $w$ given by 
TABLE I. Reduced partition function $z_{N}$ for $N=2,3, \ldots, 10$.

$z_{2}=b\left(3+b^{2}\right)$
$z_{3}=b^{2}\left(3+10 b+6 b^{2}+6 b^{3}+7 b^{4}+3 b^{5}+b^{7}\right)$
$z_{4}=b^{3}\left[1+15 b+27 b^{2}+62 b^{3}+63 b^{4}+87 b^{5}+80 b^{6}+87 b^{7}+O\left(b^{8}\right)\right]$
$z_{5}=b^{5}\left[6+37 b+105 b^{2}+231 b^{3}+413 b^{4}+669 b^{5}+921 b^{6}+1197 b^{7}+O\left(b^{8}\right)\right]$
$z_{6}=b^{7}\left[15+75 b+290 b^{2}+687 b^{3}+1590 b^{4}+2994 b^{5}+5304 b^{6}+8388 b^{7}+O\left(b^{8}\right)\right]$
$z_{7}=b^{9}\left[20+135 b+543 b^{2}+1645 b^{3}+4206 b^{4}+9381 b^{5}+19131 b^{6}+35802 b^{7}+O\left(b^{8}\right)\right]$
$z_{8}=b^{11}\left[15+173 b+780 b^{2}+2871 b^{3}+8296 b^{4}+21453 b^{5}+49110 b^{6}+104723 b^{7}+O\left(b^{8}\right)\right]$
$z_{9}=b^{13}\left[6+135 b+847 b^{2}+3612 b^{3}+12348 b^{4}+36166 b^{5}+93972 b^{6}+223572 b^{7}+O\left(b^{8}\right)\right]$
$z_{10}=b^{15}\left[1+57 b+615 b^{2}+3261 b^{3}+13503 b^{4}+45345 b^{5}+134610 b^{6}+357933 b^{7}+O\left(b^{8}\right)\right]$

$$
w=\sqrt{\Omega^{2}-N \omega^{2}},
$$

which means that the frequency $\Omega$ of the center of mass is larger than the frequencies $w$ of the degrees of freedom in the relative coordinate system. Changing the sign of $\omega^{2}$ allows us to obtain the case with $w$ larger than $\Omega$.

In our path-integral treatment presented in [1], a recurrence relation was obtained for the partition function $\mathbb{Z}_{I}(N)$ corresponding to the degrees of freedom with frequency $w$ in the relative-coordinate system. Introducing

$$
b=e^{-\beta \hbar w}
$$

for brevity in the notation, we found that

$$
\mathbb{Z}_{I}(N)=\frac{1}{N} \sum_{m=0}^{N-1} \xi^{N-m-1}\left(\frac{b^{(N-m) / 2}}{1-b^{N-m}}\right)^{3} Z_{I}(m) .
$$

This recurrence relation applies for bosons $(\xi=+1)$ and for fermions $(\xi=-1)$. The subscript $I$ refers to identical particles, which can be specified to be fermions (subscript $F$ ) or bosons (subscript $B$ ). The total partition function $Z_{I}(N)$ differs from $\mathbb{Z}_{I}(N)$ only by a factor that accounts for the centerof-mass contribution

$$
Z_{I}(N)=\left(\frac{\sinh \frac{1}{2} \beta w}{\sinh \frac{1}{2} \beta \Omega}\right)^{3} Z_{I}(N)
$$

\section{B. The "sign" problem and the canonical ensemble}

For three-dimensional fermions, the contribution (2.4) to the partition function from the relative degrees of freedom clearly illustrates the kind of numerical inaccuracies that originate for the fermion case $\xi=-1$. If the partition functions for $1,2, \ldots,(N-1)$ particles are known, Cramer's rule can be used to calculate the partition function for $N$ fermions. Factorizing the denominators in the partition function (2.4) by introducing the quantities

$$
z_{N}=b^{-3 N / 2} Z_{F}(N) \prod_{j=1}^{N}\left(1-b^{j}\right)^{3},
$$

a careful analysis shows that $z_{N}$ are polynomials in $b$. Typical terms of the expansion in powers of $b$ are summarized in Table I. In Table II the polynomial $z_{10}$ is given in full detail.

These expressions clearly illustrate that the recurrence relation (2.4) with its alternating signs is numerically not

TABLE II. Reduced partition function $z_{10}$.

$$
\begin{aligned}
& z_{10} / b^{15}=1+57 b+615 b^{2}+3261 b^{3}+13503 b^{4}+45345 b^{5}+134610 b^{6}+357933 b^{7}+879054 b^{8}+2010684 b^{9}+4345128 b^{10} \\
& +8918028 b^{11}+17522121 b^{12}+33074766 b^{13}+60269475 b^{14}+106291845 b^{15}+182005221 b^{16}+303159450 b^{17} \\
& +492298273 b^{18}+780509769 b^{19}+1210116969 b^{20}+1836796808 b^{21}+2732828889 b^{22}+3989023158 b^{23} \\
& +5717909554 b^{24}+8054427489 b^{25}+11158011888 b^{26}+15210615846 b^{27}+20416394163 b^{28}+26995546500 b^{29} \\
& +35180518626 b^{30}+45204591771 b^{31}+57294327336 b^{32}+71652967164 b^{33}+88450188705 b^{34}+107801980392 b^{35} \\
& +129760468767 b^{36}+154293502560 b^{37}+181278676665 b^{38}+210486919309 b^{39}+241585183659 b^{40} \\
& +274128160656 b^{41}+307573138975 b^{42}+341282238438 b^{43}+374550513570 b^{44}+406618287529 b^{45} \\
& +436710170877 b^{46}+464053823241 b^{47}+487924429070 b^{48}+507663537606 b^{49}+522721627332 b^{50} \\
& +532669844520 b^{51}+537233278185 b^{52}+536289729798 b^{53}+529888599492 b^{54}+518233866459 b^{55} \\
& +501687264528 b^{56}+480736747215 b^{57}+455986553010 b^{58}+428116140777 b^{59}+397862850832 b^{60} \\
& +365978330982 b^{61}+333210742599 b^{62}+300265798386 b^{63}+267794502825 b^{64}+236364132672 b^{65} \\
& +206455118098 b^{66}+178444248222 b^{67}+152611340019 b^{68}+129133921739 b^{69}+108101637948 b^{70} \\
& +89519477403 b^{71}+73326342790 b^{72}+59402845476 b^{73}+47590266765 b^{74}+37699218057 b^{75}+29525967669 b^{76} \\
& +22859393796 b^{77}+17493002580 b^{78}+13228933857 b^{79}+9885384267 b^{80}+7297628572 b^{81}+5321514726 b^{82} \\
& +3832246167 b^{83}+2725103340 b^{84}+1912972224 b^{85}+1325507922 b^{86}+906297026 b^{87}+611414355 b^{88} \\
& +406837455 b^{89}+266998784 b^{90}+172746528 b^{91}+110189817 b^{92}+69257169 b^{93}+42900069 b^{94}+26170152 b^{95} \\
& +15728664 b^{96}+9303894 b^{97}+5421180 b^{98}+3106746 b^{99}+1753755 b^{100}+972819 b^{101}+531755 b^{102} \\
& +285204 b^{103}+150903 b^{104}+78197 b^{105}+40038 b^{106}+20010 b^{107}+9928 b^{108}+4758 b^{109}+2298 b^{110} \\
& +1056 b^{111}+492 b^{112}+213 b^{113}+99 b^{114}+39 b^{115}+18 b^{116}+7 b^{117}+3 b^{118}+b^{120}
\end{aligned}
$$


stable: The leading terms are of order $b^{M}$, where $M$ increases drastically if the number of fermions increases. Nevertheless, the expression for $z_{10}$, e.g., is useful to check expressions for the partition function or derived quantities for their accuracy. Because for the fermions, unlike the boson case, solving the recurrence relations thus runs into severe numerical problems, we will use the generating function technique for the actual calculation of the free energy and the internal energy. To convince ourselves that numerical inaccuracies have been avoided, the internal energy of the model for up to ten fermions has been calculated both ways, i.e., from the recurrence relations and with the generating function technique, and the results of both methods coincide. How the chemical potential and the internal energy are calculated will be elaborated in the next section.

\section{THERMODYNAMIC PROPERTIES}

The generating function $\Xi_{F}(u)$ corresponding to the partition functions $\mathbb{Z}_{F}(N)$ is defined in the standard way as

$$
\Xi_{F}(u)=\sum_{N=0}^{\infty} u^{N} Z_{F}(N)
$$

As shown in [1], it is given by

$$
\Xi_{F}(u)=\exp \left(-\sum_{j=1}^{\infty} \frac{1}{j} \frac{\left(-u b^{3 / 2}\right)^{j}}{\left(1-b^{j}\right)^{3}}\right)
$$

This means that in our model the internal degrees of freedom are represented by a system of noninteracting oscillators with frequency $w . \Xi_{F}(u)$ is then formally the grand-canonical partition function of that subsystem. However, it is not the grand-canonical partition function of the full model system with interaction for two reasons: First, one has to take the center-of-mass correction into account and, second, the eigenfrequency $w$ in the relative coordinate system depends on the number of particles. However, given $w$, the full mechanism of the generating functions is applicable in the relative coordinate system provided afterward the necessary center-of-mass corrections are taken into account.

The partition function $\mathbb{Z}_{F}(N)$ from the internal degrees of freedom can be obtained by inverting the defining Taylor series (3.1),

$$
Z_{F}(N)=\frac{1}{2 \pi i} \oint_{C} \frac{\Xi_{F}(z)}{z^{N+1}} d z
$$

where $C$ is a closed contour in the complex $z$ plane around the origin. The generating function $\Xi_{F}(z)$ is inaccessible for numerical purposes. However, considering a circular contour with radius $u$, one obtains

$$
\begin{aligned}
\mathbb{Z}_{F}(N) & =\frac{1}{2 \pi} \int_{0}^{2 \pi} \frac{\Xi_{F}\left(u e^{i \theta}\right)}{u^{N}} e^{-i N \theta} d \theta \\
& =\frac{1}{2 \pi} \int_{0}^{2 \pi} \exp \left[\ln \Xi_{F}\left(u e^{i \theta}\right)-N \ln u\right] e^{-i N \theta} d \theta .
\end{aligned}
$$

The extremum of $\left[\ln \Xi_{F}\left(u e^{i \theta}\right)-N \ln u\right]$ on the real axis satisfies the condition $N=u(d / d u) \ln \Xi_{F}(u)$. Using Eq. (3.2), this requirement becomes

$$
N=\sum_{\nu=0}^{\infty} n_{\nu}, \quad n_{\nu}=\frac{1}{2} \frac{(\nu+1)(\nu+2)}{1+e^{-\beta\left(\mu-\epsilon_{\nu}\right)}}, \quad \epsilon_{\nu}=\hbar w\left(\nu+\frac{3}{2}\right),
$$

which is precisely the result that one would obtain from the grand-canonical treatment with $u=e^{\beta \mu}$, taking into account the degeneracy $\frac{1}{2}(\nu+1)(\nu+2)$ of the $\nu$ th energy level. Factorizing out the steepest-descent contribution $\Xi_{F}(u) / u^{N}$ obtained this way, one finds

$$
\begin{gathered}
\mathbb{Z}_{F}(N)=\frac{\Xi_{F}(u)}{u^{N}} \int_{0}^{\pi} \Psi(\theta) d \theta, \\
\Psi(\theta)=\frac{1}{\pi} \frac{\Xi_{F}\left(u e^{i \theta}\right)}{\Xi_{F}(u)} e^{-i N \theta},
\end{gathered}
$$

where $\Psi(\theta)$ is a real function, suitable for numerical integration if $u=e^{\beta \mu}$ is determined. The advantage of a procedure based on the generating function is that all contributions to $\Xi_{F}(u)$ turn out to be positive, in contrast to the direct determination of the partition function (2.4), which numerically involves severe sign problems, as argued in Sec. II.

\section{A. The chemical potential}

The chemical potential has to be determined from the requirement (3.5). There are clearly two cases to be considered. For sufficiently low temperature, $\mu$ will be larger than $\frac{3}{2} \hbar w$, but at high temperature $\mu$ might be smaller than $\frac{3}{2} \hbar w$.

For the case $\mu>\frac{3}{2} \hbar w$, the behavior of the denominator is fundamentally different for the energy levels $\epsilon_{\nu}<\mu$ as compared to those satisfying $\epsilon_{\nu}>\mu$. Assume that $\mu$ will be found in the interval $] \epsilon_{L-1}, \epsilon_{L}$ ] between two consecutive levels

$$
\mu=\epsilon_{L}-\hbar w \alpha, \quad \alpha \in[0,1[,
$$

and treat the levels $\epsilon_{\nu} \leqslant \mu$ separately from those with $\epsilon_{\nu}$ $>\mu$. We start the discussion under the assumption $L \geqslant 1$ and rewrite $N$ as

$$
\begin{aligned}
N= & \sum_{\nu=1}^{L} \frac{1}{2} \frac{(L-\nu+1)(L-\nu+2)}{1+b^{-\alpha+\nu}}+\frac{1}{2} \frac{(L+1)(L+2) b^{\alpha}}{1+b^{\alpha}} \\
& +\sum_{\nu=1}^{\infty} \frac{1}{2} \frac{(L+\nu+1)(L+\nu+2) b^{\alpha+\nu}}{1+b^{\alpha+\nu}} .
\end{aligned}
$$

Let $N_{L}$ denote the (not necessarily integer) value that the right-hand side of this equation would take for $\alpha=0$, i.e., if 


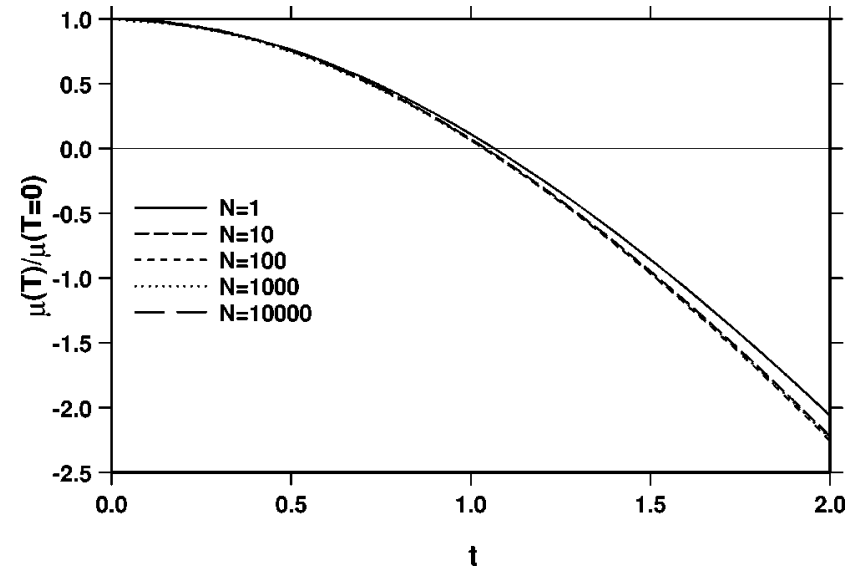

FIG. 1. Scaled chemical potential $\mu(T) / \mu(T=0)$ as a function of the scaled temperature $t=k T / \hbar w N^{1 / 3}$ for $10,100,1000$, and 10000 fermions. For reference, this quantity is also plotted for one particle.

the chemical potential would be located exactly at $\epsilon_{L}$. One finds after some algebra (provided $L>0$ )

$$
\begin{aligned}
N_{L}= & \frac{1}{6}\left(L+\frac{3}{2}\right)(L+2)(L+1)+(2 L+3) \sum_{\nu=1}^{L} \frac{\nu b^{\nu}}{1+b^{\nu}} \\
& +\sum_{\nu=L+1}^{\infty} \frac{1}{2} \frac{(L+\nu+1)(L+\nu+2) b^{\nu}}{1+b^{\nu}}>\frac{1}{6} L^{3} .
\end{aligned}
$$

The result $L<\left(6 N_{L}\right)^{1 / 3}$ is important for developing a surefire algorithm to find the chemical potential. If one starts from a value $L$ that is the largest integer smaller than $(6 N)^{1 / 3}$, it implies that $\mu<\epsilon_{L}$. One can then decrease the value of $L$ step by step, until a value of $L$ is found for which $N_{L} \geqslant N$ but $N_{L-1}<N$.

Fortunately, this procedure can also be used if $\mu$ is smaller than the lowest energy of the model. It then results in a negative value of $L$, which is related to the chemical potential in Eq. (3.8) by formally filling out $\epsilon_{L}$ with $L$ negative.

A fresh refining routine is then started to find $\alpha \in[0,1[$, which is bracketed as required for sure-fire root-finding programs. The actual determination of $\alpha$ (for both cases $\mu$ $>\frac{3}{2} \hbar w$ and $\left.\mu<\frac{3}{2} \hbar w\right)$ is straightforward from the equation for $N$ if written in the appropriate form for numerical treatment:

$$
\begin{aligned}
N= & \sum_{\nu=0}^{\infty} \frac{1}{2} \frac{(\nu+1)(\nu+2)}{1+b^{L-\nu-\alpha}}=\sum_{\nu=0}^{\infty} \frac{1}{2}(\nu+1)(\nu+2) \\
& \times \begin{cases}\frac{1}{1+b^{L-\nu-\alpha}} & \text { for } \nu \leqslant L-\alpha \\
\frac{b^{-L+\nu+\alpha}}{b^{-L+\nu+\alpha}+1} & \text { otherwise. }\end{cases}
\end{aligned}
$$

The determination of the chemical potential along these lines presents no numerical difficulties. Because the Fermi energy is of order $\hbar w(6 N)^{1 / 3}$, it seems natural to express $k T$ in units of $\hbar w N^{1 / 3}$. This scaling factor turns out to be surprisingly good, as is shown in Fig. 1, where the temperature dependence of the chemical potential $\mu(T)$ in units of $\mu(0)$ is plotted against $t=k T / \hbar w N^{1 / 3}$. Introducing a density of states and making the continuum approximation $\left(k_{B} T\right.$ $\ll \hbar w)$, the corresponding scaling law $[15,16]$ with the Fermi energy is implicit.

\section{B. The free energy}

Having determined the chemical potential, the free energy $\mathbb{F}_{F}(N)=-(1 / \beta) \ln \mathbb{Z}_{F}(N)$ can be evaluated from Eq. (3.6),

$$
\begin{gathered}
\mathbb{F}_{F}(N)=\mathbb{F}_{F}^{(0)}(N)-\frac{1}{\beta} \ln \left(\int_{0}^{\pi} \Psi(\theta) d \theta\right), \\
\mathbb{F}_{F}^{(0)}(N)=-\frac{1}{\beta} \ln \frac{\Xi_{F}(u)}{u^{N}},
\end{gathered}
$$

where $\mathbb{F}_{F}^{(0)}(N)$ is the zeroth-order steepest-descent result, which would be obtained from the grand-canonical treatment. The correction factor involving $\Psi(\theta)$ accounts for the finite number of particles. For reference, $\Psi(\theta)$ is shown for $N=10$ in Fig. 2 as a function of $\theta$ for various values of the temperature. If $N$ increases, $\Psi(\theta)$ becomes increasingly concentrated near the origin $\theta=0$ for nonzero temperatures.

The resulting free energy per particle as a function of temperature is shown in Figs. 3, 4, and 5 for $N=1, N=10$, and $N=100$, respectively, in units of $\hbar w(6 N)^{1 / 3}$ proportional to the Fermi energy. For comparison, the contribution $\mathbb{F}_{F}^{(0)}(N)$ is also plotted (dashed lines). As expected, this steepest-descent contribution becomes increasingly accurate if the number of fermions increases.

\section{The internal energy}

The contribution of the relative degrees of freedom to the internal energy

$$
\mathbb{U}_{F}=\frac{d}{d \beta}\left(\beta \mathbb{F}_{F}\right)=\mathbb{F}_{F}-T \frac{d}{d T} \mathbb{F}_{F}
$$

can be obtained from the free energy obtained above by numerical differentiation. The internal energy per particle is plotted in Fig. 6 in units of $\hbar w(6 N)^{1 / 3}$ proportional to the Fermi energy. A scaling law similar to that for the chemical potential is observed.

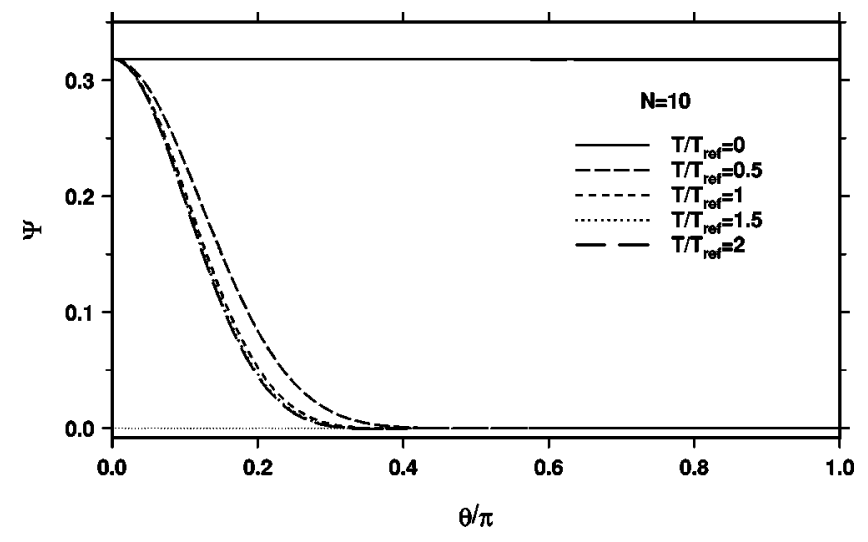

FIG. 2. Integrand $\Psi(\theta)$ of Eq. (3.7) for ten fermions as a function of $\theta$ for various values of the temperature, expressed in units of $T_{\text {ref }}=\hbar w N^{1 / 3} / k$. 


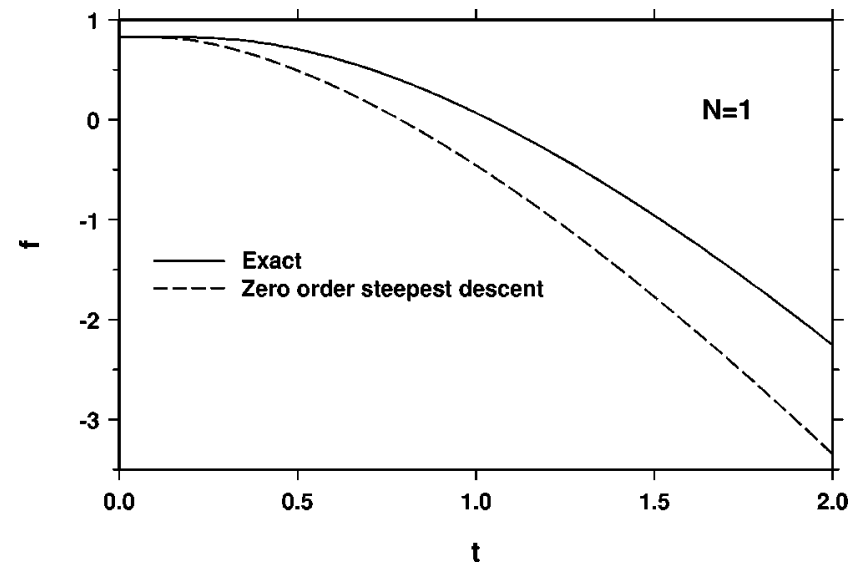

FIG. 3. Scaled free energy per particle $f=\left(\mathbb{F}_{F} / N\right) / \hbar w(6 N)^{1 / 3}$ as a function of the scaled temperature $t=k T / \hbar w N^{1 / 3}$ for 1 particle. For comparison, the zeroth-order steepest-descent contribution is also plotted (dashed line).

In one dimension the thermodynamical properties of harmonically interacting bosons and fermions can be derived from each other. This case has been studied in [1]. In higher dimensions, the fermion internal energy is smoothly decreasing with decreasing temperature, whereas for the boson case it shows sudden changes in slope, related to the condensation.

To within the numerical accuracy, the results are in agreement with the standard description from the generating function treatment using

$$
\sum_{\nu=0}^{\infty} \epsilon_{\nu} n_{\nu}=\hbar w \sum_{\nu=0}^{\infty} \frac{1}{2} \frac{(\nu+1)(\nu+2)(\nu+3 / 2)}{1+e^{-\beta\left(\mu-\epsilon_{\nu}\right)}} .
$$

These results can also be compared with the internal energy $\mathrm{U}_{F, \text { rec }}(N)$, which one would obtain from the recurrence relation. In terms of the expressions for $z_{N}$ discussed above, one then obtains

$$
\mathrm{U}_{F, \text { rec }}(N)=\hbar w\left(\frac{b}{z_{N}} \frac{\partial z_{N}}{\partial b}+\frac{3}{2} N+3 \sum_{j=1}^{N} \frac{j b^{j}}{1-b^{j}}\right) .
$$

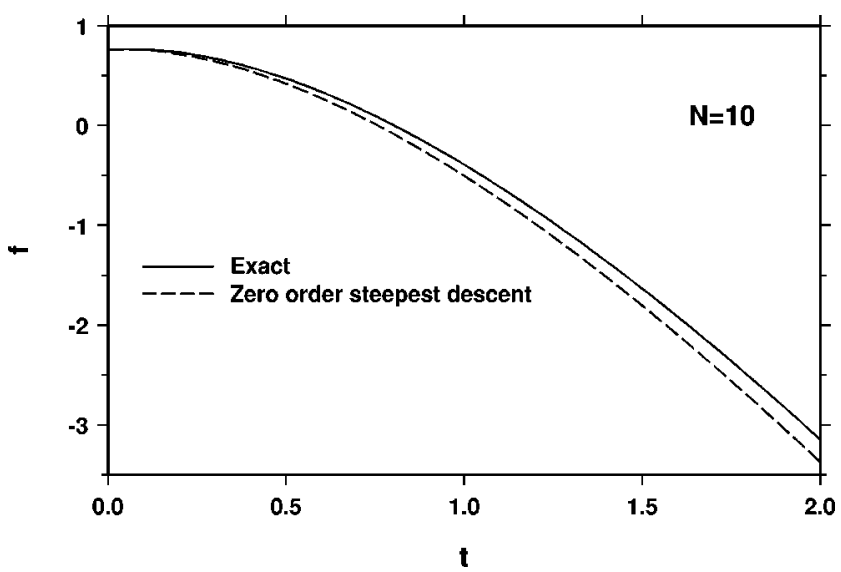

FIG. 4. Same as Fig. 3, but for ten fermions.

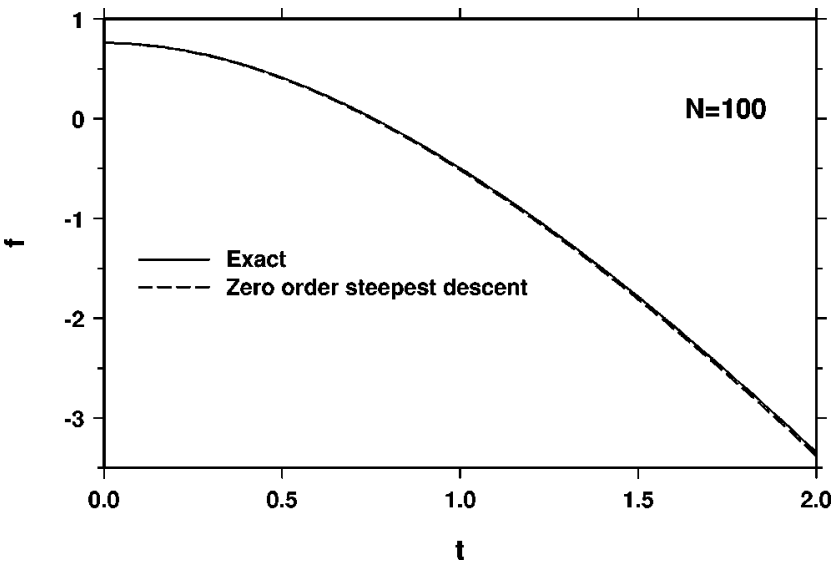

FIG. 5. Same as Figs. 3 and 4, but for 100 fermions.

This calculation is in practice only feasible for a limited number of particles $N \leqslant 10$ and for these cases it coincides within the numerical accuracy with the results plotted in Fig. 6.

\section{GROUND-STATE ENERGY}

In this section the low-temperature limit will be considered. By counting the number of occupied energy levels, the dominant contribution to the partition function can then be calculated easily, taking into account the degeneracy $\frac{1}{2}(\nu$ $+1)(\nu+2)$ of the levels with energy $\epsilon_{\nu}=\hbar w\left(\nu+\frac{3}{2}\right)$. The calculation is done first with the Fermi level $L$ fully occupied. The number of particles $N_{F}$ required for this assumption to hold is

$$
N_{F}=\sum_{\nu=0}^{L} \frac{1}{2}(\nu+1)(\nu+2)=\frac{1}{6}(L+3)(L+2)(L+1) .
$$

Consequently, the Fermi energy is of order $(6 N)^{1 / 3} \hbar w$. The total energy $U_{F}$ associated with the case with the $L$ th level fully occupied is

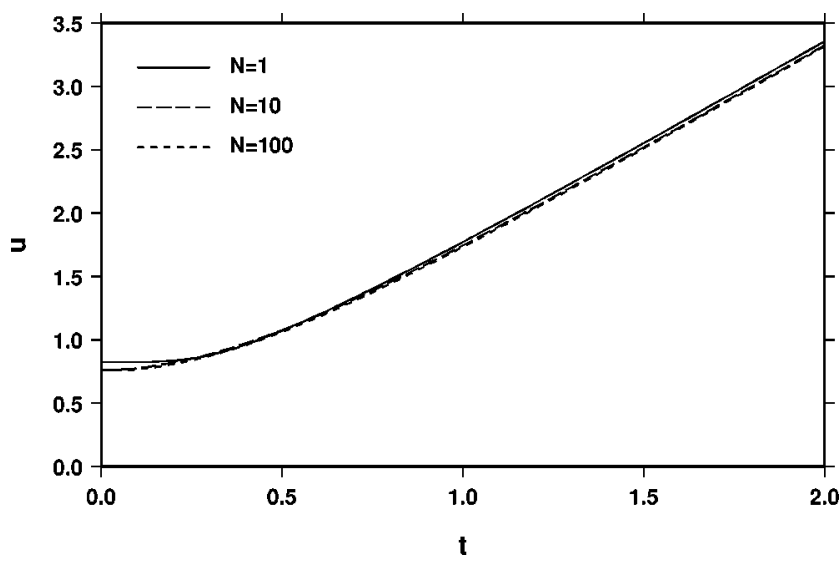

FIG. 6. Scaled internal energy per particle $u$ $=\left(\mathrm{U}_{F} / N\right) / \hbar w(6 N)^{1 / 3}$ as a function of the scaled temperature $t$ $=k T / \hbar w N^{1 / 3}$ for 10 and 100 fermions. For reference, the result for one particle is also plotted. 
TABLE III. Number of particles $N_{F}$ and total energy $U_{F}$ with the energy level $\nu_{F}$ fully occupied.

\begin{tabular}{rrrrrcrrc}
\hline \hline$\nu_{F}$ & $N_{F}$ & $U_{F} / \hbar w$ & $\nu_{F}$ & \multicolumn{1}{c}{$N_{F}$} & $U_{F} / \hbar w$ & $\nu_{F}$ & \multicolumn{1}{c}{$N_{F}$} & $U_{F} / \hbar w$ \\
\hline 1 & 4 & 9 & 10 & 286 & 2574 & 60 & 39711 & $\frac{3693123}{2}$ \\
2 & 10 & 30 & 15 & 816 & 10404 & 70 & 62196 & 3358584 \\
3 & 20 & 75 & 20 & 1771 & $\frac{58443}{2}$ & 80 & 91881 & $\frac{11301363}{2}$ \\
4 & 35 & $\frac{315}{2}$ & 25 & 3276 & 66339 & 90 & 129766 & 8953854 \\
5 & 56 & 294 & 30 & 5456 & 130944 & 100 & 176851 & $\frac{27058203}{2}$ \\
6 & 84 & 504 & 35 & 8436 & 234099 & 150 & 585276 & 66721464 \\
7 & 120 & 810 & 40 & 12341 & $\frac{777483}{2}$ & 200 & 1373701 & $\frac{416231403}{2}$ \\
8 & 165 & $\frac{2475}{2}$ & 45 & 17296 & 609684 & 250 & 2667126 & 504086814 \\
9 & 220 & 1815 & 50 & 23426 & 913614 & 300 & 4590551 & $\frac{2079519603}{2}$ \\
10 & 286 & 2574 & 55 & 30856 & 1319094 & 400 & 10827401 & $\frac{6528922803}{2}$ \\
\hline \hline
\end{tabular}

$$
\begin{aligned}
\mathrm{U}_{F} & =\sum_{\nu=0}^{L} \frac{1}{2}(\nu+1)(\nu+2) \hbar w\left(\nu+\frac{3}{2}\right) \\
& =\frac{1}{8} \hbar w(L+3)(L+1)(L+2)^{2} .
\end{aligned}
$$

For a limited number of particles, the number of particles and the energy $U_{F}$ are shown in Table III.

For an arbitrary number $N$ of fermions not filling the Fermi level completely, the determination of the groundstate energy is slightly more involved. We first determine the number of particles $N_{F} \leqslant N$ that fill the level $L$. From Eq. (4.1) it follows that the highest fully occupied level $L$ is given by

$$
\begin{aligned}
L= & \operatorname{int}\left[\left(3 N+\frac{1}{9} \sqrt{3^{6} N^{2}-3}\right)^{1 / 3}\right. \\
& \left.+\frac{1}{3}\left(3 N+\frac{1}{9} \sqrt{3^{6} N^{2}-3}\right)^{-1 / 3}-2\right] .
\end{aligned}
$$

From this level $L$, one can determine the corresponding $N_{F}$ and $\mathrm{U}_{F}$.

The resulting formula for the leading term in the partition function for $N$ particles in the zero-temperature limit is $b^{3 N / 2} b^{M_{N}}$, which defines the power $M_{N}$ as

$$
M_{N}=(L+1)\left(N-\frac{1}{24}(L+2)(L+3)(L+4)\right) \text {. }
$$

The ground-state energy $E_{0}$ with $N$ particles is $E_{0}=\mathrm{U}_{F}$ $+\left(N-N_{F}\right) \hbar w\left(L+1+\frac{3}{2}\right)$ because the remaining particles are in the level $L+1$ and consequently

$$
\begin{aligned}
E_{0}= & -\frac{1}{24} \hbar w(L+4)(L+3)(L+2)(L+1) \\
& +N \hbar w\left(L+1+\frac{3}{2}\right) .
\end{aligned}
$$

\section{CONCLUSION AND DISCUSSION}

In this paper we have given a short review of the calculation techniques for fermions, described in [1] and [2] for identical particles in general. Next a numerical analysis of the chemical potential and of the free energy is made for a given expectation value of the number of particles as a function of temperature. In this analysis, we could easily illustrate what the consequences are of the minus sign coming from the antisymmetric representation of the permutation group in the expression for the partition function. Even when the expressions are known analytically, the plot of a relatively smooth function such as the free energy requires special techniques as a consequence of numerical instabilities due to a sign problem in the recurrence relation for the partition function. The necessity of such techniques can be checked by attempting a calculation of a few limits, which lead to the application of l'Hôpital's rule many times, even proportional to the square of the number of particles in the system.

It should be noted that the model contains only spinpolarized fermions. In quantum dots, its production would require a magnetic field. In our model this field is not included. However, as we have shown in [1], the expressions for the partition function for fermions in the presence of an external magnetic field can be obtained with the same calculational technique. The influence of the magnetic field on the chemical potential and the specific heat of our model has not been studied yet for fermions. In alkali-metal vapors the spin polarization of the fermions would be inherent to the experimental technique [20].

The chemical potential and the internal energy exhibit a scaling law in the sense that it is an almost universal function of the temperature when plotted in the indicated scaled units. Although we strongly suspect that the scaling comes via the Fermi level of the confined system, as is the case in the continuum limit, we have no mathematical proof of this observation in the low-temperature case $k_{B} T \ll \hbar w$.

We did not compare the present approach with other theories using the same or an analogous model. It should be stressed, however, that we presented here results obtained with a different scheme for the evaluation of path integrals for fermions.

\section{ACKNOWLEDGMENTS}

A discussion with W. Krauth on related topics is gratefully acknowledged. This work is performed in the frame- 
work of the FWO Projects Nos. 1.5.729.94, G.0287.95, and WO.073.94N (Wetenschappelijke Onderzoeksgemeenschap, Scientific Research Community of the FWO on "Low-Dimensional Systems"); the Interuniversity Poles of Attraction Program, Belgian State, Prime Minister's
Office, Federal Office for Scientific, Technical and Cultural Affairs, and in the framework of the BOF NOI projects of the University of Antwerpen. One of the authors (F.B.) thanks the FWO for financial support.
[1] F. Brosens, J. T. Devreese, and L. F. Lemmens, Phys. Rev. E 55, 227 (1997).

[2] F. Brosens, J. T. Devreese, and L. F. Lemmens, Phys. Rev. E 55, 6795 (1997).

[3] S. Stringari, Phys. Rev. Lett. 76, 1405 (1996).

[4] F. Brosens, J. T. Devreese, and L. F. Lemmens, Phys. Rev. A 55, 2453 (1997).

[5] M. H. Anderson, J. R. Ensher, M. R. Matthews, C. E. Wieman, and E. A. Cornell, Science 269, 198 (1995).

[6] K. B. Davis, M. O. Mewes, M. R. Andrews, N. J. van Druten, D. S. Durfee, D. M. Kurn, and W. Ketterle, Phys. Rev. Lett. 75, 3969 (1995).

[7] C. C. Bradlet, C. A. Sacket, J. J. Tollett, and R. G. Hulet, Phys. Rev. Lett. 75, 1687 (1995).

[8] S. Grossman and M. Holthaus, Z. Naturforsch. Teil A 50A, 323 (1995); 50A, 921 (1995).

[9] S. Grossman and M. Holthaus, Phys. Lett. A 208, 188 (1995).
[10] W. Ketterle and N. J. van Druten, Phys. Rev. A 54, 656 (1996).

[11] K. Kirsten and D. J. Toms, Phys. Rev. A 54, 4188 (1996).

[12] H. Haugerud, T. Haugset, and F. Ravndal, Phys. Lett. A 225 , 18 (1997).

[13] L. Cohen and C. Lee, J. Math. Phys. 26, 3105 (1985).

[14] W. Krauth, Phys. Rev. Lett. 77, 3695 (1996).

[15] D. A. Butts and D. S. Rokhsar, Phys. Rev. A 55, 4346 (1997).

[16] I. F. Silvera and J. T. M. Walraven, J. Appl. Phys. 52, 2304 (1981).

[17] L. F. Lemmens, F. Brosens, and J. T. Devreese, Phys. Lett. A 189, 437 (1994).

[18] F. Brosens, J. T. Devreese, and L. F. Lemmens, Solid State Commun. 96, 137 (1995).

[19] L. F. Lemmens, F. Brosens, and J. T. Devreese, Phys. Rev. E 53, 4467 (1996).

[20] H. T. C. Stoof, M. Houbiers, C. A. Sackett, and R. Hulet, Phys. Rev. Lett. 75, 3969 (1995). 